\title{
The component of traditional Hakka residential architecture decoration and its cultural spirit
}

\author{
Liwu Zeng \\ Jiaying university school of Fine Arts, China
}

\begin{abstract}
Keywords: Hakka traditional dwellings; decorative elements; landscape painting; teaching exploration.
\end{abstract}

\begin{abstract}
At the landscape painting time of guiding students to appreciate the beauty of Hakka traditional architectural decorative, through recording and discovering decorative creation art from dwelling ancestors, mining the spirit of traditional culture and education function which contains, we will extract essence and give characteristics in inheritance to develop it.
\end{abstract}

\section{Introduction}

Traditional Hakka folk house originate in the Central Plains residential buildings in the historical society, in the influence of Historical factors and natural conditions, it uses mountain building materials to built building group for family life and have defensive function. In the Hakka traditional residential buildings, a building decoration component combines utility and beauty, which contains lots of information, which is the crystallization of hundreds of years of life to understand the Hakka people. Meizhou-a gathering place of Hakka culture, it has a great of art painting resource. In landscape painting course, at the same time of guides the student to appreciate the beauty of the Hakka traditional architectural decorative component, we will through recording, discovery, understanding of dwelling ancestors creation, mining the spirit of traditional culture and education function which contains, we will extract essence and give characteristics in inheritance to develop it.

\section{The characteristics of traditional residential architecture component of Hakka}

The Hakka people great importance in residential decorative component modeling, in their view, residential construction is a start of hundred years and the decisive factor of the impact of future development prosperity of the whole family, the decorative arts of Hakka residential endowed strong national spirit and aesthetic taste and means, which reflects the rich cultural connotation of the Hakka people; Hakka people in accordance with the laws of beauty to beautify life, reconstruction of life, enhance the spice of life, but also with the help of these decorative elements to express good wishes and sentiment, which reflects the wishes and the prospect of people family harmony, the lucky and happy, health and longevity, make one's ancestors illustrious.

Outdoor uses stone, brick, gray plastic, porcelain and other rain proof material, and more rugged simplicity of expression to decoration and mold far view decorative component, both in subject matter, color and materials technology, it has highlights of aesthetically pleasing decorative effect. Is the most representative of the Hakka building is gate, it represents the people of vision for the future and the source of confidence for the future. In color processing, commonly used red, black, green and other colors of Its brightness dazzles the eyes to point decoration, making the gatehouse broke the simple sense of color and increased sense of three-dimensional surface, and Hakka gatehouse have decorated the special cultural connotation, such as: a town of evil with the lion, decorative elements on the pillars in the gatehouse implication rich peony, meaning look very happy magpie plum, Aquarius moral security and peace, and some with the story of the "loyalty" and "filial piety" "courage" "righteousness" characters wood components, and so on, therefore, some decoration component of Hakka architecture deeply reflects the Hakka people's world outlook and outlook on life, enriching the cultural characteristics ( Figure 1). 


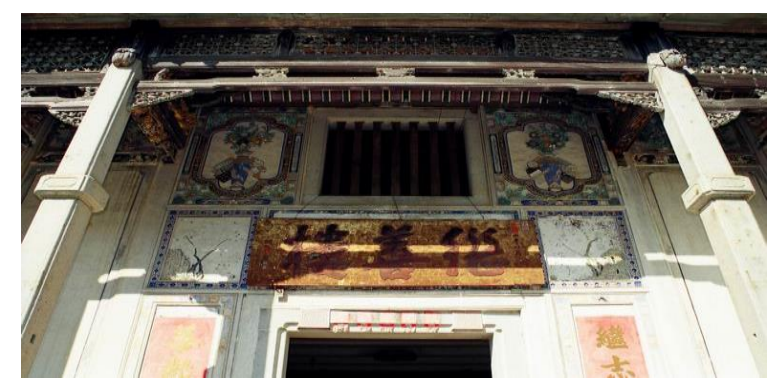

Fig. 1 Hakka architecture cultural characteristics

Relative outdoor decoration component properties, wood carving is the main component of Hakka architecture interior decoration. Wood component use carving, relief, hollow above on the beam, column, window, screen, railing, incense table ,decorated with flowers, landscape, birds and insects, dragon and Phoenix, kylin reached the effect of practical, appreciation, moral. Such as Meizhou round dragon house Chengde floor, its decorative component of building is very rich, beam column gate decorated with wood carvings, kylin doors on both sides of each pair, and then with plum, luck and other decorative form, there are four fan pierced with relief and use of large screen door. The main theme is a vase, flower decoration. Hall after hall wall carved shrines, screen door, side hall carved wooden bar. Its characteristic is vigorous, rough lines, lively style, and color quaint, strong and supple. Hollowed out relief and use, it is tight structure, strong decoration. Unity construction phase of the overall style of decoration, stable, durable and practical function, it has certain representative in the traditional Hakka residential decoration (Figure 2).

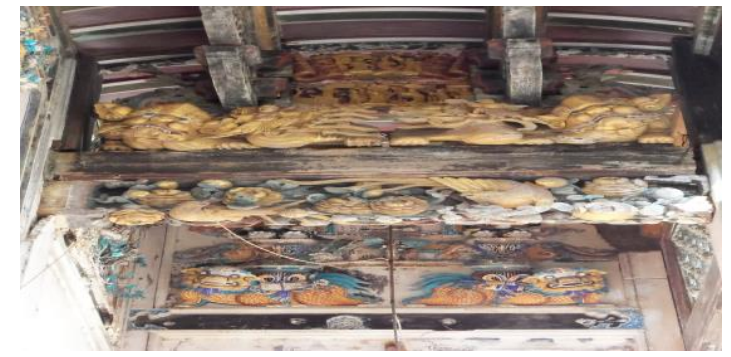

Fig. 2 the traditional Hakka residential decoration

\section{Exploration in the teaching of landscape painting in the education of traditional culture spirit of decorative elements of traditional dwellings of Hakka contains}

Landscape painting has a unique superiority in the aspect of training sense of color and expression ability, at the same time, it plays an essential role in training students on the distribution of color pictures from the whole and design capability, which directly affect the students in painting or design on color and color layout using ability. But the traditional culture in the modern education plays a role that can not be ignored. Therefore, leading students to landscape painting not only can highlight the superiority of landscape painting, but also can through the traditional culture education to improve the quality of students. Manifest objects and decorative elements of traditional dwellings of Hakka Hakka culture has the spirit, it is meticulous and contains fine moral. That requests us must put the record, found, inheritance, development role into education in teaching ideas in landscape sketch teaching.

Landscape painting comes from recording. At the same time of recording beauty, as far as possible the more system, more complete.

Good records in addition to pay attention to the sketch of the spots and lines and the arts knowledge skill, it should make the record function more system, complete, the way that to reach is carefully observe. As in the beam of the sketch, you'll find out Hakka houses not only in beam structure, but also in decorative frame. The class of beam structure is refers to bear and transfer, it forms the backbone of housing, composing of columns, beams, Fang, Tan, brackets, melon columns. Decoration class beam of Hakka houses has the integration of unique regional style. Decoration class 
beam is with the same to tan, general layout in the tan bar that below the space, it will play definition role of space and beautify the. A lot of Tulou hall beam decorative wood carving, lions lying, Fei, lotus, loose bract etc. Such as the lions that lying Yongkang floor beam and side beam between, they are not a matter of whitewash, but is gentle and lovely; The bearing cover that hall prior to the corridor connected with the outer ring, the bearing cover eaves of Liang Zhumu architecture ornate, under the roof of melon and beam column connection point, having ornaments mosaic hollowed out, at the lower of a column next to the hanging columns, carved with carved with lotus seat. The inner ring of the exquisite craft between the Zhenfu each have change. Some decorations also are coated with thick and heavy colors. Such as Kui hall decorated floor beam, bright color, colorful; Up to the eaves brackets hanging carved loose core, lotus, gourd, carving delicate, and the whole building has 8 pairs of. Table, column of Rong Lu first hall carved with dragon and Phoenix, birds, animals and flowers, with different expression.

At the time of Landscape painting recording, we will find and exploration of traditional spiritual of Hakka culture. This includes full of decorative elements found in the culture and beautiful meaning, it finds ancestors of life, art of refining technique.

Found the source of creative inspiration, mining ancestors. Beauty is a more common than personality. As architectural decoration in the component of various flowers, birds, grass, wood, animal, fetish can take into the decoration, they are beauty, moral, practical ,decorated and interesting, even on is aesthetic education for the next generation influence. From these decorative elements that created from ancestors, we can be seen that the Hakka people are advocating natural, they thought of harmony and unity is the highest realm. For their physical and mental health, for future generations of the rich, even if there is no green mountains and rivers, they will build the Fengshui woods behind the house, and built a pond in front of the house, trees, garden, pond beautiful picturesque landscape to achieve the purpose that the nature harmonious and unified. In the processing of art that children have, the understanding of the United States and is integrated, continuous.

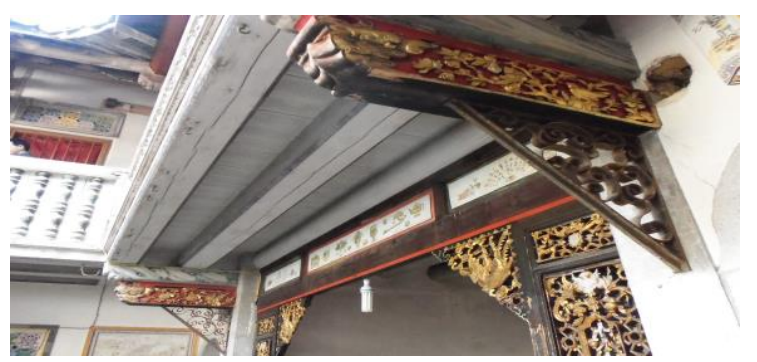

Fig. 3 Meixian Yan Yang Qiao Xi Jishan building

The research on continuity of decorative elements of traditional dwellings Hakka should be combined with the new process in landscape sketch teaching, and comply with the aesthetic requirements of the times, it will be inheritance in the tradition, it is full integration of traditional and modern techniques of expression, rolling in undertaking the inheritance and development to promote innovation.

Traditional decorative elements do not hinder the application in construction of main function's improvement. This requires the inheritance in the development, if not from the innovation life and the cultural inheritance, it is like water without a source, a tree without roots. Beauty is a kind of feeling, even can only be sensed imagery, and culture is long-term, the impact is far-reaching. Landscape painting can assume the inheritance and innovation of the primary promoter function. Let the students perception of traditional culture, traditional spirit makes the students perceived to convey the importance of understanding through the Hakka decorative elements, In the Hakka traditional residence teaching, students can get a feeling of beauty in the imperceptibly, and purified in the spirit. That is to say, the influence of aesthetic education in art produced not by rigid instillation and forced to receive, but art contains the beauty and significance of edification, infected recipient results. Works 
of art have a specific and vivid perceptual image to express the author's thoughts and feeling, which means that the receiver must be use its beauty and the means to feel works with whole heart of power input, with the same breath, because only in this way, the receiver can feel all the charm of the works. In this case, the recipient will be direct communication works like there may not be any reflection and conscious memory to interrupt and barrier themselves. It is the understanding of the Hakka building decorative elements in landscape painting process, students are imperceptibly by the works of infection and education, their spiritual realm will change imperceptibly. It can be said, this "influence character by environment" of art aesthetic education and the role of features like gentle to the seedlings and grow sturdily, although run silently.

In summary, decorative elements of traditional dwellings of Hakka both have aesthetic psychological habits of the Chinese tradition, and form a relatively clear "Hakka" feature, the characteristics of many art shows are heavy example to our comprehensive understanding of Hakka people's aesthetic ideas. While these characteristics will be transformation different because of space and time, but the understanding of fusion is the basis of art evolution. The human pursuit of the good development is endless landscape, and the simple record and imitation is not the purpose of education. In the face of rich traditional of Hakka decoration component, as educators, let the student in the recording of Hakka architecture decoration component and the beautiful in landscape sketch teaching, making them fully understand the spirit of traditional culture, the bridge and promoters of inheritance is the requirements for scenery to write their own improve living, also is the topic that educators must carefully explore.

\section{Reference}

[1]YangSanghehe. The Hakka architecture and protection of cultural heritage [J]. The perspective of landscape anthropology. Academic research, 2013 (4).

[2] Lin Aifang. On the application of "Hakka woodcarving art in the traditional residential buildings [J]. Journal of Jiaying University (PHILOSOPHY AND SOCIAL SCIENCES), 2005 (4).

[3]Wang Hongjian, Yuan Baolin. An introduction of art. [M]. Higher education press, 1994 (7). 